\title{
Ad Serving Using a Compact Allocation Plan
}

\author{
Peiji Chen \\ Yahoo!Labs \\ peiji@yahoo-inc.com
}

Chandrashekhar
Nagarajan

Yahoollabs

cn54@yahoo-inc.com

Erik Vee
Yahoo!Labs
erikvee@yahoo-inc.com

\author{
Wenjing Ma \\ Yahoo!Labs \\ wenjingm@yahoo- \\ inc.com \\ Jayavel \\ Shanmugasundaram \\ Google* \\ jayavel.shanmugasundaram@acm.org \\ Manfai Yu
Yahoo!Labs
manfai@yahoo-inc.com
}

\begin{abstract}
A large fraction of online display advertising is sold via guaranteed contracts: a publisher guarantees to the advertiser a certain number of user visits satisfying the targeting predicates of the contract. The publisher is then tasked with solving the ad serving problem - given a user visit, which of the thousands of matching contracts should be displayed, so that by the expiration time every contract has obtained the requisite number of user visits. The challenges of the problem come from (1) the sheer size of the problem being solved, with tens of thousands of contracts and billions of user visits, (2) the unpredictability of user behavior, since these contracts are sold months ahead of time, when only a forecast of user visits is available and (3) the minute amount of resources available online, as an ad server must respond with a matching contract in a fraction of a second.

We present a solution to the guaranteed delivery ad serving problem using compact allocation plans. These plans, computed offline, can be efficiently queried by the ad server during an ad call; they are small, using only $O(1)$ space for contract; and are stateless, allowing for distributed serving without any central coordination. We evaluate this approach on a real set of user visits and guaranteed contracts and show that the compact allocation plans are an effective way of solving the guaranteed delivery ad serving problem.
\end{abstract}

\section{INTRODUCTION}

A central problem facing online advertising systems is ad serving, i.e., deciding how to rapidly match supply (user visits) and demand (ads) in a way that meets an overall objective. Our focus in this paper is on guaranteed delivery display advertising, a multi-billion dollar industry, whereby advertisers can buy a fixed number of targeted user visits (e.g., 100 million user visits by Males in California visiting Sports web pages) for a future duration (e.g. July-August 2011), and internet publishers guarantee these visits months in advance. In such cases, when the user visits (supply) actually occur, the publisher's ad server is faced with a splitsecond decision of choosing an appropriate guaranteed contract (demand) in such a way that in the aggregate, all the

\footnotetext{
*Work was done while with Yahoo! Labs
}

guarantees are met. In addition, as part of the split-second decision, the ad server also needs to ensure some secondary objectives such as uniformly serving user visits to guaranteed contracts; e.g., if there is a guaranteed contract that spans two months, the advertiser expects their ad to be shown throughout the duration, rather than just being shown to user visits that occur during the first few days.

The above scenario can be modeled as an assignment problem. We are presented with a set of user visits $I$, a set of guaranteed contracts $J$, and a set of feasible allocation edges $E \subseteq I \times J$, which indicates which user visits are eligible to be served by which guaranteed contracts. Each guaranteed contract $j \in J$ has a demand $d_{j}$, and we are asked to allocate the user visits to satisfy the demands. In addition, there is an objective function over the feasible allocations, such as uniform delivery over the duration of the contracts, and the goal is to find the allocation that maximizes the objective function.

Solving the above problem even in an offline setting presents a formidable set of challenges. First, the set $I$ is extremely large, approaching tens of billions of user visits every day for large publishers; since we may have to solve the problem for up to a year in advance in the case of guaranteed contracts, this results in an effective set size of tens to hundreds of trillions! (Note that the set of edges $E \subseteq I \times J$ is even larger!) Second, we often do not know the set $I$ exactly, but only approximately; for instance, we do not know precisely which users will visit a particular web page, but only have a stochastic estimate of the set based on past history.

Of course, our actual situation is even more challenging because the ad server needs to solve the above problem online, usually within a hundred milliseconds per request, in order to meet the latencies for end-user facing actions such as page views. Further, in order to meet the throughput requirements, the ad server needs to solve the online problem in parallel among hundreds to thousands of distributed machines that do not communicate directly with each other for each user visit, while still ensuring that the online decisions attempt to maximize the overall objective. Finally, due to the long-tailed nature of user behavior, the ad server needs to explicitly deal with the fact that it may encounter user visits that have not been seen before, but must nevertheless 
ensure that the online decisions attempt to maximize the overall objective. These are a daunting set of issues - what do we do?

One simple approach is to design an ad server that selects a contract based on how many impressions (i.e. user visits) have been previously served to each eligible contract. For instance, if there are two competing contracts, one of which is severely under-delivering and the other which is delivering well (or even slightly over-delivering), the ad server may decide to serve the under-delivering contract. While this approach is conceptually simple and practical (in fact, it serves as the baseline in our experiments), it has a number of subtle problems. First, the above approach requires a good definition of the notion of "under-delivering." The most straightforward method is simply to assume that contracts should be served at a uniform rate - a contract with demand of 30 million impressions and a duration of 30 days should deliver about 1 million impressions a day. This has the advantage of making delivery smooth, when possible. But it ignores the fact that the actual traffic varies greatly over time. Weekends have less traffic than weekdays, while the traffic at night is typically much smaller than the traffic during the day. In addition, there may be an upcoming dearth of impressions available for contracts, which may require the ad server to deviate from the uniform rate in order to meet the guarantee. This can occur, for instance, when some type of user visits are promised exclusively for premium contracts at some time in the future (e.g., a sports company buys out all visits to Yahoo! Super Bowl pages); this may require the ad server to deliver more than the uniform rate during the days before the event in order to meet guarantees. Second, using the above approach, the ad servers need to maintain some notion of how much a contract has delivered in (near) real-time so that they can compute under-delivery at any point in time. In cases where there are thousands of ad servers distributed across the world, this problem becomes quite challenging, especially when user visits exhibit certain locality patterns that cause non-uniform traffic to be sent to different ad servers.

Another approach Vee et al. 2010] that addresses many of the above issues is to solve an allocation problem offline, and then send a rate-based compact allocation plan to the distributed ad servers. The allocation plan is based on a forecast of user visits over the lifetime of the contracts, so it effectively addresses the issue of what it means for a contract to under-deliver. (It is when it does not meet the goal at the end of its lifetime, even if it is above or below the uniform serving rate currently). Further, for a certain class of objectives, the allocation plan is rate-based, meaning that the ad server does not rely on any online counts of how much a contract has served in the immediate past, but is based on a serving probability that does not require online state Vee et al. 2010. However, this approach also has a set of issues that need to be addressed. First, this approach is very sensitive to the accuracy of the forecast of user visits. For instance, if the forecast for a certain set of user visits is twice a large, then the the serving rate will be half as large as it should be. Second, this approach typically requires solving a non-linear convex optimization problem, which can have on the order of billions of variables, which can be quite expensive in terms of time and resources.

\subsection{Contributions}

In order to address the above issues with ad serving, we propose the following two-part solution. First, we introduce a novel, lightweight algorithm for solving the offline allocation problem. The algorithm, which we call High Water Mark (HWM), produces a compact allocation plan, requiring $\mathrm{O}(1)$ numbers per contract. Further, it is stateless and rate-based, which ensures that multiple ad servers can execute the algorithm in parallel without any bottlenecks. Although it does not have the strong theoretical guarantees of Vee et al. 2010, it has the advantage of quick optimization. (Finding an allocation plan takes only a few minutes, even with a billion arcs in the demand-supply forecast graph.) Second, we introduce a rapid feedback loop from the ad server to the optimization system, which reacts to recent aggregate delivery statistics in order to correct for any supply forecasting errors. In fact, we can even provide theoretical bounds on how the frequency of feedback enables the HWM algorithm to correct for supply forecasting errors (these bounds are also borne out experimentally). Thus, the feedback loop, coupled with the quick HWM optimization, enables the ad serving system to quickly react to unforeseen effects, while still effectively addressing supply and demand constraints that vary over the duration of the contracts.

In addition, we briefly describe an implementation of a variant of the algorithm of Vee et al. 2010 as a point of comparison, which we call the DUAL algorithm.

We present a thorough evaluation of the HWM algorithm, comparing it to a real world baseline which is currently responsible for serving a large fraction of Yahoo!'s guaranteed contracts. Unlike the HWM algorithm, which relies on forecasts of user supply to make allocation decisions, the baseline algorithm is a well-tuned feedback based approach. At a very high level, it increases the serving rate for the underdelivering contracts, and, vice-versa, decreases it for those serving too aggressively. We show that the proposed algorithm greatly improves overall delivery of the contracts, while maintain a smooth serving rate.

We further present experiments comparing HWM and DUAL. We see that the two perform effectively the same- the under-delivery for HWM is on par with DUAL, and its smoothness is actually better. Thus, HWM is a good choice in practice, due to its lightweight implementation and quick optimization cycle time.

\subsection{Paper Outline}

We formally define the guaranteed delivery allocation problem in Section 2 and present an overview and the system architecture in Section 3 We state the proposed HWM offline algorithm along with the online HWM allocation algorithms in Section 4. In Section 5 we describe our experiment and metrics. In Section 6, we thoroughly evaluate the proposed approach against a real world production baseline. Then, in Section 7, we compare HWM and DUAL. We review the related work in Section 8 and conclude with some open problems in Section 9.

\section{MODEL AND PROBLEM STATEMENT}

We begin by providing a detailed example of the guaranteed delivery display advertising problem. Recall that we are presented with a set of advertisers, each aiming to reach a specific number of user visits (contract demand) that fall under the targeting parameters.

Consider the problem pictorially represented in Figure 1 


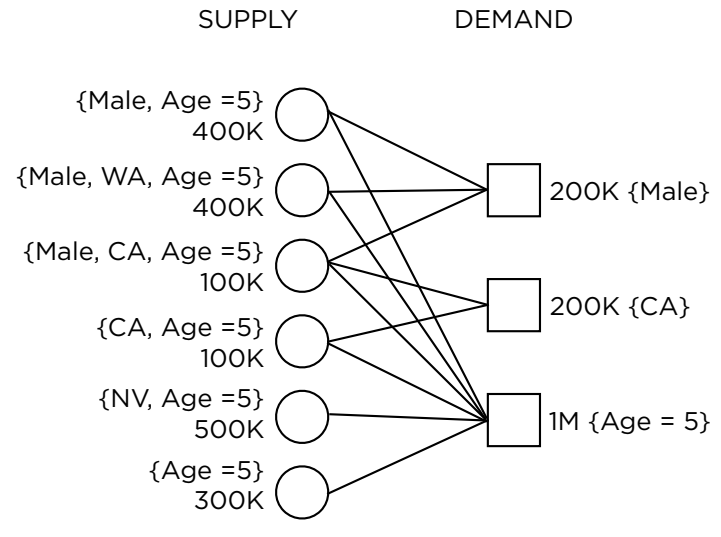

Figure 1: An example allocation problem. Each user (left) is connected to the contracts that it is eligible for (right).

There are three advertisers, targeting Males, users from California, and users with Age in the 5th bucket. There are 6 different kinds of users, all of them satisfying the age constraint. Some of the users are known to be from California, Washington or Nevada; while for others their location is unknown. Similarly, some the publisher knows are male, while for others the gender is unknown. Each node on the supply side is annotated with the total forecasted number of visits, for example we expect to see 100,000 user visits from Californians with age in the 5th bucket and unknown gender. On the demand side each contract is annotated with the total number of user visits guaranteed to it by the publisher. An edge between a supply node $i$ and a demand node $j$ indicates that supply $i$ is eligible for $j$, that is it satisfies all of the targeting criteria.

The ad server must find an allocation of each supply node to the demand. For example, to satisfy the second contract both the third (Male, CA, Age = 5) and the fourth (CA, Age $=5$ ) nodes must be fully allocated to the second contract. When a Male user from California with Age $=5$ arrives it is eligible for all three of the contracts. However, it must be allocated to the second contract; otherwise the second contract will not reach its desired demand and the publisher will be hit with underdelivery penalties.

We note that while the above example is a small illustration, the actual problem is much bigger in at least three dimensions. First, the number of user visits is of the order of billions per day, and guaranteed contracts can be sold several months in advance. Second, the number of attributes per user visit is of the order of hundreds, including demographic information such as age, gender, location, explicit interest, inferred interests, recent browsing behavior, and so on; page information such as the topic and ad-size; and time information including date, user time and global time. Finally, the number of guaranteed contracts is of the order of tens to hundreds of thousands for large publishers. Consequently, the scale of the graph - both in terms of supply and demand - is quite large. Also, in practice, the advertiser targeting constraints are complex boolean expressions on the attributes of the supply.

\subsection{Problem Statement}

Let $I$ be the set of [forecasted] user visits, and $J$ be the set of contracts. We model the allocation problem as a bipartite graph $G=(I \cup J, E)$ where there is an edge $(i, j) \in E$ if the user visit $i$ can be used to satisfy contract $j$. The bipartite graph encodes the contention between contracts, for example, a node $j \in J$ with few outgoing edges means that it is a finely targeted contract, and there are few users satisfying the targeting constraints. Conversely, a node $i \in I$ with a high degree means that it is a highly contended user, that is there are many contracts that wish to advertise to this particular user.

In addition to the targeting constraints, each contract $j \in$ $J$ specifies its desired demand $d_{j}$. Likewise, a user may have multiple identical visits, so each node $i \in I$ is labeled with the total supply, $s_{i}$.

We wish to find a feasible allocation, that is a value on each edge $x_{i j}$, representing the fraction of supply $i$ is allocated to contract $j$. We say that $x$ is a feasible solution if it satisfies:

$$
\begin{array}{lr}
\forall_{j} \quad \sum_{i \in \Gamma(j)} x_{i j} s_{i} \geq d_{j} & \text { demand constraints } \\
\forall_{i}, \quad \sum_{j \in \Gamma(i)} x_{i j} \leq 1 & \text { supply constraints } \\
\forall_{(i, j) \in E}, \quad x_{i j} \geq 0 & \text { non-negativity constraints, }
\end{array}
$$

where following standard notation, $\Gamma(i)$ are the neighbors of $i: \Gamma(i)=\{j:(i, j) \in E\}$, and similarly for $\Gamma(j)$.

Although we do not explicitly require it, one important aspect of serving is smoothness of delivery. Advertisers do not want their entire demand to be served in a single hour (nor do users!), even if this technical satisfies the demand. Thus, any serving method should roughly serve an equal number of impression each day for a given contract, subject to natural variations in contention, sell-through rate, and overall traffic patterns.

There are, of course, potentially many additional secondary considerations. For a longer description of the formal model and possible competing objectives of the offline optimization system see Yang and Tomlin 2008, Bharadwaj et al. 2010 Ghosh et al. 2009 Alaei et al. 2010.

\section{SOLUTION OVERVIEW AND SYSTEM ARCHITECTURE}

Before laying out our solution, we first discuss several alternatives.

Perhaps the simplest ad serving solution is to randomly flip an unbiased coin between all the matching contracts. This solution is fast, space-efficient, has a large throughput, requires no coordination, and generalizes to previously unseen input. Unfortunately, what it gains in speed and flexibility, it loses in accuracy. Indeed, because this approach makes no distinction between contracts during contract selection, it will typically lead to severe under-delivery and thus loss of revenue. For instance, in the example in Figure 1. all Males in CA in Age Group 5 should be allocated to the second contract even though it is eligible for all three contracts, or the second contract will under-deliver.

An alternative solution that tries to maximize accuracy is as follows. Solve the allocation problem offline, and remember the allocation values $x_{i j}$ on all the arcs of the bipartite graphs in the ad server machines. Then, given a user visit, the ad server can simply look up the allocation and assign it to the appropriate contract. While this would certainly 


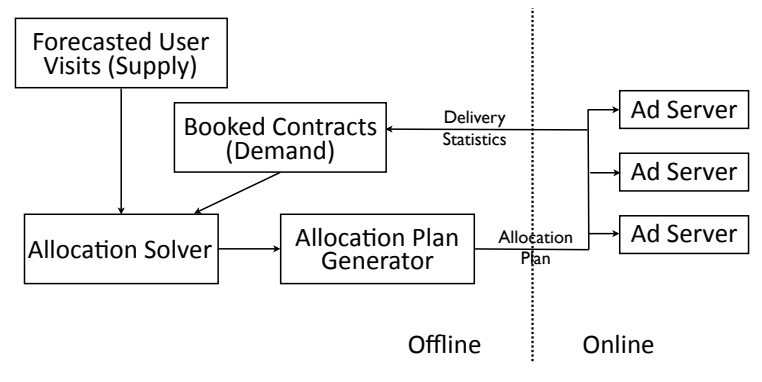

Figure 2: Proposed System Architecture

be accurate (it implements the optimal allocation), it is very memory intensive given the large number of nodes and edges that need to be stored. Further, and perhaps more importantly, it is not generalizable. Specifically, it is impractical to assume that we can predict all the possible user visit types that could possibly occur (recall that it is a combination of dynamic user characteristics such as interests, along with their IP location, pages they visit, etc.) and solve an optimization problem for all such visits. As an illustration, consider again the example in Figure 1, and a Female user from California with Age $=5$. Since this type of user was not considered in computing the offline allocation, there are no $x_{i j}$ values present. Therefore, while there are two eligible contracts, the ad server has no information as to which contract should be shown. In this example we would prefer to show the "CA" contract, since it is harder to satisfy. However, this information is not easy to elicit from the $x_{i j}$ values.

Intuitively, we want to get the best of both approaches, obtaining the accuracy of the second approach, while retaining the simplicity and scalability of the first approach. In Section 4 we present the overview of a solution that achieves these goals.

\subsection{System Architecture}

Figure 2 shows the proposed system architecture. As shown, there is an offline component called the Allocation Plan Generator that takes in a forecast of user visits and booked contracts, and solves the allocation problem. We solve the problem on a sample of user visits, which makes computing the offline solution of the problem fast and practical. The resulting allocation plan is sent to all the ad server machines.

Each ad server machine loads up the allocation plan at start-up time, and stores the plan in memory. Then, whenever it needs to process a user visit, it first finds the matching contracts, and then uses the allocation plan to select a contract to show. Since the latter phase is local (it only involves the user visit and its matching contracts), there is no need for the ad server machines to communicate with each other during serving, nor is there any need for an ad server machine to maintain global state or even local counters!

Further, as shown in the figure, there is a feedback loop from the Ad Server to the Booked Contract Demand. Specifically, the demand of a contract is adjusted to account for the actual delivered impressions. Since there can be errors in the forecast, the Allocation Plan Generator is re-run periodically to correct for any forecast errors based on this actual remaining demand and a new allocation plan is then distributed to the ad servers.

In the subsequent section of the paper, we elaborate on the Allocation Plan Generator and the Ad Server components in more detail. (A more detailed discussion of the User Visit Forecasting system is beyond the scope of this paper. See Agarwal et al. 2010 for a description of one such possible system.)

\section{RATE-BASED ALGORITHMS}

In this section, we describe two rate-based serving algorithms. The first is the High Water Mark Algorithm (HWM). The second is an implementation of the dual-based method of Vee et al. 2010, which we will refer to as DUAL.

Both algorithms involve two phases. The offline phase, described in Section 4.1 takes as input the demand-supply forecast graph and produces an allocation plan. This allocation plan is just a few numbers per contract, independent of the number of impressions. The online phase, described in Section 4.1.1. repeatedly takes as input a user visit and the set of eligible contracts for that user visit, and decides which ad to serve based on the allocation plan.

The HWM algorithm is lightweight and fast, even in the offline phase. The DUAL algorithm, while lightweight and fast during the online phase, is not nearly as fast in the offline phase. (In our experience, it is more than an order of magnitude slower.) However, it has the advantage of yielding provably optimal allocation during serving, assuming that forecasts are perfect. Of course, forecasts are far from perfect in practice. Thus, the benefits of allocation using DUAL are somewhat mitigated.

The key to having a compact allocation plan is having a way to infer the the $x_{i j}$ values at serving time without explicitly storing them. This can be done by creating an $\alpha_{j}$ fraction which represents the fraction of demand to be give to the contract, and which is the same for all neighboring supply nodes of a contract. The mathematical basis of this representation comes from the fact that an optimal solution can be represented using these $\alpha_{j}$ values Vee et al. 2010. Although Linear Programming solvers can be used to find an optimal solution, due to the size of the problem and time constraints, we describe a much faster heuristic.

\subsection{HWM Algorithm}

Offline optimization for HWM uses a simple, but surprisingly effective heuristic for generating the allocation plan. The algorithm generates a serving rate for each contract $j$, denoted $\alpha_{j}$, together with an allocation order. The allocation order is set so that a contract $j$ with less eligible supply (i.e. $\sum_{i \in \Gamma(j)} s_{i}$ ) has higher priority than those with more eligible supply. By labeling each contract with just two numbers, the HWM algorithm creates a compact and robust allocation plan.

During online serving, every contract gets an $\alpha_{j}$ fraction of every impression, unless this would mean giving more than $100 \%$ of the impression away. In this case, ties are broken in allocation order: a contract $j$ coming first in the allocation order will get its $\alpha_{j}$ fraction, while a contract $j^{\prime}$ coming later will get whatever fraction is left, up to $\alpha_{j^{\prime}}$ (possibly getting nothing for some impressions).

The algorithm itself labels every contract $j$ with its eligible supply, denoted $S_{j}$, which is used to determine the allocation order; contracts with smaller $S_{j}$ values come earlier in the 


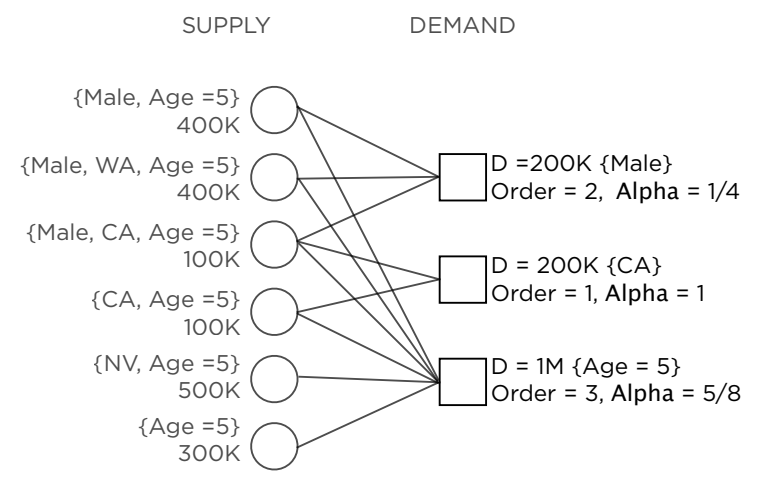

Figure 3: An example compact allocation plan with the complete supply-demand graph. Note that only the data on the RHS of the figure is sent to the ad servers.

allocation order. In order to determine the serving rate, $\alpha_{j}$ for each $j$, the HWM algorithm does the following steps.

1. Initialize remaining supply $r_{i}=s_{i}$ for all $i$.

2. For each contract $j$, in allocation order, do:

(a) Solve the following for $\alpha_{j}$ :

$$
\sum_{i \in \Gamma(j)} \min \left\{r_{i}, s_{i} \alpha_{j}\right\}=d_{j},
$$

setting $\alpha_{j}=1$ if there is no solution.

(b) Update $r_{i}=r_{i}-\min \left\{r_{i}, s_{i} \alpha_{j}\right\}$ for all $i \in \Gamma(j)$.

Notice that it is a simple manner to choose the allocation order using a different heuristic. In our experience, the available supply works quite well. Contracts that have very little time left will also have very little supply and naturally become high priority. Likewise, highly targeted contracts also tend to have much less inventory. This generally means they have less flexibility in which user visits they can be served, and they are treated with higher priority. Contrast this with a large, untargeted contract that can easily forego certain types of users visits when other highly targeted (and generally more expensive) contracts may need those visits in order to satisfy their demands.

The compact allocation plan produced by the HWM algorithm is shown in Figure 3 Note that only the right hand side of the graph is sent to the Ad Servers. In this example the allocation plan results in a feasible solution. We note that the allocation plan is highly dependent on the supply forecast, and an incorrect supply forecast may yield suboptimal results. We will explore this issue in detail in Section 4.3

\subsubsection{Online Evaluation for HWM}

Given an allocation plan, which consists of the allocation order and serving rate $\alpha_{j}$ for each contract $j$, the role of the ad server is to select one of the matching contracts. We present the algorithm that the ad server follows below:

1. Given an impression $i$, let $J=\left\{c_{1}, c_{2}, \ldots, c_{|J|}\right\}$ be the set of matching contracts listed in the allocation order.
2. If $\sum_{j=1}^{|J|} \alpha_{j}>1$, let $\ell$ be the maximum value so that $\sum_{j=1}^{\ell} \alpha_{j} \leq 1$. Finally, let $\alpha_{\ell+1}^{\prime}=1-\sum_{j=1}^{\ell} \alpha_{j}$. Note that by definition of $\ell, \alpha_{\ell+1}^{\prime}<\alpha_{\ell+1}$.

3. Select a contract $j \in[1, \ell]$ with probability $\alpha_{j}$ and the contract $\ell+1$ with probability $\alpha_{\ell+1}^{\prime}$. Note that in the case that $\sum_{j=1}^{|J|} \alpha_{j}<1$ with some probability no contract is selected.

Consider the running example from Figure 3 In the case an impression of type $\{\mathrm{CA}$, Age $=5\}$ arrives, $\ell=1$ and the middle contract with allocation order 1 is always selected, since its serving rate is 1 . On the other hand, suppose an impression of type $\{$ Male, Age $=5\}$ arrives. Then with probability $1 / 4$ it is allocated to contract 1 , and with probability $5 / 8$ it is allocated to contract 3 , with the remaining probability $(1 / 8)$ it is left unallocated. (In case no contract is selected, the impression is then auctioned off in the Non-Guaranteed Marketplace.)

\subsection{The DUAL Algorithm}

The DUAL algorithm replaces the demand constraints described in Section 2.1 with the following:

$$
\forall_{j} \quad \sum_{i \in \Gamma(j)} x_{i j} s_{i}+u_{j} \geq d_{j}
$$

where we think of $u_{j}$ as the under-delivery for contract $j$. We additionally add the constraint that $u_{j} \geq 0$. Our objective is then

$$
\text { Minimize } \sum_{j} \sum_{i \in \Gamma(j)} s_{i}\left(x_{i j}-\theta_{j}\right)^{2} / \theta_{j}+\sum_{j} p_{j} u_{j}
$$

where $\theta_{j}$ and $p_{j}$ are fixed. The first term attempts to minimize the distance of the allocation from some target, $\theta_{j}$. We set $\theta_{j}$ to be the total demand of contract $j$ divided by its total (forecasted) supply. In this way, we attempt to make the overall mix of impressions as uniform as possible. (See Yang and Tomlin 2008, Bharadwaj et al. 2010, Ghosh et al. 2009 Alaei et al. 2010 for more discussion on this.)

The second term represents the under-delivery penalty for the contracts. We set $p_{j}$ (the penalty value per impression for contract $j$ ) to be 10 for each contract. This heuristically chosen value performs well in practice.

In order to produce an allocation plan, DUAL simply reports the dual values of the modified demand constraints for an optimal solution - in practice, these are actually the duals for an approximately optimal solution. Note that there is one dual value per contract.

\subsubsection{Online Evaluation for DUAL}

For each impression, we find the set of eligible contract. This, together with the dual values for their respective demand constraints, is enough to compute the value of the primal solution on the corresponding edges (i.e. the $x_{i j}$ values) computed during the offline phase. In fact, if the set of impressions seen during serving is the same as the set of impressions used during the offline phase, this method will faithfully reproduce exactly the same allocation. Mathematically, define $g_{i j}(z)=\max \left\{0, \theta_{j}(1+z)\right\}$. For an impression $i$, let $C_{i}$ be the set of eligible contracts, and let $\alpha_{j}$ be the dual of the modified demand constraint for each $j \in C_{i}$. Then we first solve the following for $X$ :

$$
\sum_{j \in C_{i}} g_{i j}\left(\alpha_{j}-X\right)=1 .
$$

Then, we set $\beta=\max \{0, X\}$. (It turns out that $\beta$ is the dual value for the supply constraint for impression $i$.) Finally, we 
Table 1: Increasing serving rate due to supply forecast errors

\begin{tabular}{|l|c|c|c|c|}
\hline Day & $\begin{array}{c}\text { Predicted } \\
\text { Supply }\end{array}$ & $\begin{array}{c}\text { Remaining } \\
\text { Demand }\end{array}$ & $\begin{array}{c}\text { Serving } \\
\text { Rate }\end{array}$ & $\begin{array}{c}\text { Actual } \\
\text { Delivery }\end{array}$ \\
\hline 1 & $5 \mathrm{M}$ & $2.5 \mathrm{M}$ & 0.50 & $0.4 \mathrm{M}$ \\
2 & $4 \mathrm{M}$ & $2.1 \mathrm{M}$ & 0.525 & $0.42 \mathrm{M}$ \\
3 & $3 \mathrm{M}$ & $1.68 \mathrm{M}$ & 0.56 & $0.45 \mathrm{M}$ \\
4 & $2 \mathrm{M}$ & $1.23 \mathrm{M}$ & 0.62 & $0.49 \mathrm{M}$ \\
5 & $1 \mathrm{M}$ & $0.74 \mathrm{M}$ & 0.74 & $0.59 \mathrm{M}$ \\
6 & & $0.15 \mathrm{M}$ & & \\
\hline
\end{tabular}

set $x_{i j}=g_{i j}\left(\alpha_{j}-\beta\right)$ for all $j \in C_{i}$; note that $x_{i j}=0$ for all $j \notin C_{i}$. As shown in Vee et al. 2010], this reproduces the primal solution.

As with HWM, the fractional solution is interpreted as a probability for serving. That is, contract $j$ is served to impression $i$ with probability $x_{i j}$.

\subsection{Robustness to Forecast Errors}

Since rate-based algorithms produce serving rates rather than absolute goals, it becomes much more robust to load balancing, server failures, and so on. However, it becomes more sensitive to forecast errors. For example, if the forecast is double the true value, the serving rate is half what it should be, roughly speaking. In this section, we show that in an idealized setting, frequent re-optimization mitigates this problem greatly. Note that this simple analysis applies to both HWM and DUAL.

Let us take a simple example in which our forecast is much higher than reality for all contracts. Initially, all of the contracts are underdelivering, since the actual supply is lower than the forecasted supply, the $\alpha_{j}$ given in the allocation plan is too low. As time passes, however, the rate begins to increase. This increase is not because the supply forecast errors are recognized, rather, as the expiration date for the contract approaches, the urgency with which impressions should be served to the particular contract increases.

To illustrate this point, consider a five day contract $j$ with forecasted supply of $1 M$ impressions per day, and total demand of $2.5 \mathrm{M}$ impressions. If this is the only contract in the system, we should serve it at the rate of $\alpha_{j}=2.5 \mathrm{M} / 5 \mathrm{M}=0.5$. If the supply forecasts are perfect, then the contract will finish delivering exactly at the end of the last day. On the other hand, suppose that the actual supply is only $800 \mathrm{~K}$ impressions per day (a $20 \%$ supply forecasting error rate). Then at the end of the first day the new serving rate would be recomputed to $=\alpha_{j}=2.1 M / 4 M=0.525$. Continuing with the example, we observe the behavior in Table 1, which shows that after 5 days the contract would have underdelivered by $.15 \mathrm{M} / 2.5 \mathrm{M}=6 \%$. Note that without this re-optimization, the underdelivery would be exactly $20 \%$, thus frequent reoptimizations help mitigate supply forecast errors.

We formalize the example above in the following theorem.

THEOREM 1. Suppose we have $k$ optimization cycles, the supply forecast error rate (defined to be 1 minus the ratio of real supply to predicted supply) is $r$, and the contract is never infeasible. In the case that $r>0$, the underdelivery is positive and bounded above by $\frac{r+r^{2}}{k^{1-r}}$. In the case that $r<0$, the overdelivery is positive and bounded above by $\frac{|r|}{k^{1-r}}$.
Proof. In the $i$-th round, the optimizer will allocate a $1 /(k-i+1)$ fraction of supply, of which a $(1-r)$ fraction will be delivered. Therefore, the desired demand will decrease by a factor of $1-\frac{1-r}{k-i+1}$. Therefore, the supply available in the last round (i.e. the underdelivery) is:

$$
\prod_{i=1}^{k}\left(1-\frac{1-r}{k-i+1}\right)=\prod_{i=1}^{k}\left(\frac{k-i+r}{k-i+1}\right)=\frac{r}{k} \prod_{i=1}^{k-1}\left(1+\frac{r}{i}\right)
$$

Notice that when $r>0$, this value is positive (i.e. meaning we underdeliver), while when $r<0$, this value is negative (i.e. meaning we overdeliver). First, consider the case that $r>0$. We use the fact that $\sum_{i=2}^{k-1} \frac{1}{i} \leq \ln (k-1)<\ln k$. We have

$$
\begin{aligned}
& \frac{r}{k} \prod_{i=1}^{k-1}\left(1+\frac{r}{i}\right) \leq \frac{r(1+r)}{k} \exp \left(\sum_{i=2}^{k-1} \frac{r}{i}\right) \\
& \leq \frac{r+r^{2}}{k} \exp (r \ln k)=\frac{r+r^{2}}{k^{1-r}}
\end{aligned}
$$

Now, consider the case that $r<0$. Here, we use the fact that $\sum_{i=1}^{k-1} \frac{1}{i} \geq \ln k$. Hence, we have

$$
\begin{aligned}
& \frac{|r|}{k} \prod_{i=1}^{k-1}\left(1+\frac{r}{i}\right) \leq \frac{|r|}{k} \exp \left(\sum_{i=1}^{k-1} \frac{r}{i}\right) \\
& \leq \frac{|r|}{k} \exp (r \ln k)=\frac{|r|}{k^{1-r}}
\end{aligned}
$$

So even a large $2 \mathrm{X}$ forecast error, leading to delivery rate of 0.5 of what it should be, does not result in delivering only $50 \%$ of the required demand. On a week-long contract, re-optimizing every two hours, we have $k=84$ cycles, and our under-delivery is only $8.2 \%$. Forecast errors in the other direction (which would lead to over-delivery) are even less severe. A $0.5 \mathrm{X}$ forecast error, leading to delivery rate of twice what it should be, does not result on $100 \%$ over-delivery. Instead, the over-delivery is much less than $1 \%$.

\subsection{Feedback-Based Correction}

In the previous section we showed how the supply forecast errors are mitigated by frequent reoptimization. Although this guards against large underdeliveries, the offline optimization is slow to recognize the forecast error, and the delivery rate increases very slowly in the beginning, even when faced with very large errors. An alternative solution is to employ a feedback system that would recognize the errors and correct the allocations accordingly.

Such a feedback system falls squarely in the realm of control theory, and one can imagine many feedback controllers that would achieve this task. We present a very simple protocol, which we will show performs quite well in practice. The protocol is parameterized by two variables: $\delta$, which controls the allowed slack in delivery, and a pair $(\beta+, \beta-)$ which control the boost to the serving rate.

If, during the lifetime of a contract, the contract is delivering more than $\delta$ hours behind, the feedback system increases the reported demand left for the contract by a factor of $\beta-$. For example, let $\delta=12$, and consider a 7 day contract for $70 \mathrm{M}$ impressions. Ideally, the contract should deliver $3 \mathrm{M}$ impressions by the end of day 3 . If, say, only $2 \mathrm{M}$ impressions were still not delivered by noon on day 4 , the feedback system would increase the demand left $(5 \mathrm{M}=7 \mathrm{M}-2 \mathrm{M})$ by a 


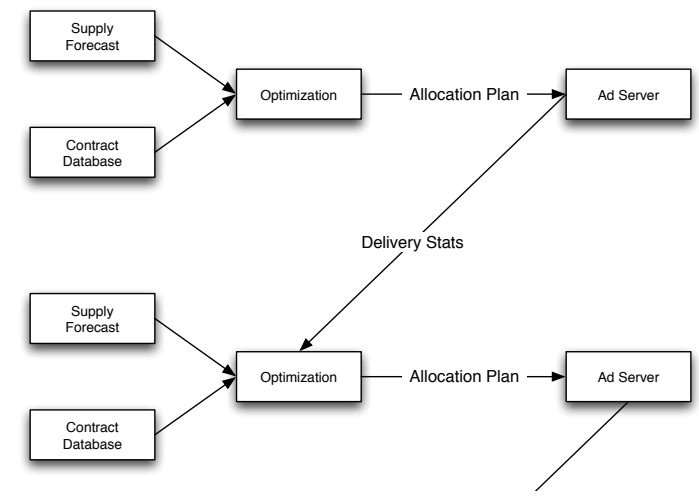

Figure 4: Experimental Setup

factor of $\beta+$, making it $5 \mathrm{M} \times \beta+$. If on the other hand, the contract had already delivered $3 \mathrm{M}$ impressions by noon on day 3 , the feedback system would decrease its demand left by a factor of $\beta-$. We will show experimentally in Section 5 that this, admittedly simple, system performs very well in real world scenarios.

\section{EXPERIMENTAL SETUP}

To validate our approach we implemented our algorithms and evaluated them using a large snapshot of real guaranteed delivery contracts and a log of two weeks of user visits. We describe the setup in detail below.

\subsection{Data flow}

Figure 4 shows the experimental setup flow. To simulate the offline procedures, we use a snapshot of guaranteed contracts stored in a contract database. A supply forecasting system using historical information produces predicted user visits matching each of the contracts in the database. The forecasted user visits along with the contracts are passed to a graph generator which generates a corresponding snapshot of the supply-demand bipartite graph. Finally, the compact allocation plan is generated as described in Section 4.1

To simulate the online environment, an ad server receives the compact allocation produced by the offline phase. We only used one ad server for each experiment since all the ad servers are completely symmetric with respect to the allocation plan they receive and the decisions they make (this symmetry is, in fact, one of the key advantages of the proposed architecture with respect to load balancing, etc.). A set of user visits recorded from real ad serving logs is then streamed through the ad server, which uses the allocation plan based reconstruction procedure detailed in Section 4 to select the appropriate contract. Finally, we record the number of user visits allocated to each contract.

\subsection{Metrics}

The main metric for ad serving is total delivery rate, defined as the total number of impression delivered, divided by the total number of impressions booked (of course for a single contract, the number of delivered impressions is bounded by its demand). Since the contracts are sold in a guaranteed fashion, underdelivery represents not only unrealized revenue (since one cannot charge for impressions not delivered), but also bad will towards the advertisers, as they typically rely on guaranteed delivery contracts for major marketing campaigns (e.g. new movies, car model launches, etc.). We will measure our results as percentage improvement in underdelivery over the baseline system. For example, if the baseline system had underdelivered on $10 \%$ of impressions, and the HWM approach underdelivered on $7 \%$, this would result in a $30 \%$ improvement.

A secondary measure of the quality of the delivery is the smoothness of the delivery. Intuitively, a week long contract should delivery about $1 / 7$ of its impressions on every day of the week; it should not deliver all of the impressions in the first day (or the last day) of the campaign. Of course, due to the interaction between different advertisers, such perfect smoothness is not always possible, however the ad server should strive to deliver impressions as smoothly as possible.

To measure smoothness formally, denote by $y_{j}(t)$ the total delivery to contract $j$ at time $t$, and $y_{j}^{*}(t)$ the optimal smooth delivery at time $t$. For example, for a week long contract for $7 \mathrm{M}$ impressions that begins at noon on Sunday, $y_{j}^{*}(t)$ would be $1 \mathrm{M}$ at noon on Monday, and $5.5 \mathrm{M}$ at 11:59PM on Friday night. We can then denote the smoothness of a contract at time $t, \sigma_{j}(t)=100 \cdot \frac{y_{j}(t)-y_{j}^{*}(t)}{d_{j}(t)}$. Finally, to reduce smoothness to a single number, let $\sigma^{f}(t)$ denote the $f$-th quantile of $\sigma_{j}(t)$ over all contracts $j$. We will typically be interested in smoothness at the 75 th and 95 th percentiles. Finally, we will report $\sigma^{f}=\max _{t} \sigma^{f}(t)$. So, for example, a smoothness of score of 4.5 at 95 th percentile means that $95 \%$ of contracts were never over-delivering by more than $4.5 \%$ compared to the optimal smooth delivery.

\section{COMPARISON OF HWM AND BASELINE}

Our first set of experiments compare a production-quality baseline to the HWM algorithm.

\subsection{Data}

We used a two week period as our simulated testing time period. From the many real contract active during that time window, we selected a representative set of 1986, which included a mix of day long, multi day and multi week long contracts; ranging from very targeted to broad, untargeted contracts.

On the impression side, we use actual ad serving logs to produce a set of real impressions for our test. For scalability, we downsample at a rate greater than $1 \%$, resulting in $20 \mathrm{M}$ impressions total. (The real traffic rate is impossible to simulate without utilizing hundreds of machines.) Even at such a high sampling rate, the full two week simulation requires about tens hours of processing to evaluate.

\subsection{Baseline}

We used the current guaranteed delivery system, named Base, as a baseline for our experiments. This is a system that aims to maintain a smooth temporal delivery on all of the contracts. At a high level, it frequently checks and increases the serving rate of contracts that are falling behind their ideal delivery numbers, and decreases the rate of those that have gotten too far ahead. The system is very robust to traffic changes and supply forecast errors, but, in contrast to the proposed HWM approach, it is reactive, rather than proactive. For example, if a certain slice of inventory is sold out in the coming days, the offline optimization will recognize the fact and may frontload a competing contract 
targeting that area. The reactive system, on the other hand, will not recognize the error until it is too late.

\subsection{Experimental Results: Regular Serving}

The experimental results are shown in Table 2. As we can see, the overall delivery is much better with the HWM approach, as the underdelivery rate falls by $53 \%$. However, the extra delivery comes at the cost of smoothness - of the $25 \%$ of contracts that frontloaded, most did so by almost 3 times the frontloading rate of Base. Recall that Base specifically targets perfectly smooth delivery. So although the smoothness of HWM here is higher than ideal, it is still not dramatically unacceptable.

Table 2: Total delivery for Base and HWM approaches. Note that HWM reduces underdelivery by $53 \%$, but has worse overall smoothness.

\begin{tabular}{|l|c|c|c|}
\hline Algorithm & Delivery Improvement & $\sigma^{75}$ & $\sigma^{95}$ \\
\hline \hline Base & - & - & - \\
HWM & $53 \%$ & $+288 \%$ & $+634 \%$ \\
\hline
\end{tabular}

\subsection{Experimental Results: Supply Forecast Er- rors}

The main culprit behind the inferior smoothness are the supply forecast errors. Recall, that the allocation plan, which determines the serving rates, $\alpha_{j}$ for each contract $j$, is computed offline using a forecast of the expected supply. In the case that the forecast is lower than the actual supply, the system will use a higher rate of serving than necessary, resulting in frontloading, that is delivering impressions ahead of the smooth linear goal. On the other hand, if the forecast is higher than actual supply, this will result in backloading, and potential underdelivery of the contracts.

The description of the supply forecasting system is beyond the scope of this work, but the distribution of errors is shown in Figure 5. (The scale of the y-axis is suppressed for confidentiality reasons.) Although many of the forecasts are accurate, we can see that a significant fraction have predictions that are inaccurate enough to potentially cause serving errors.

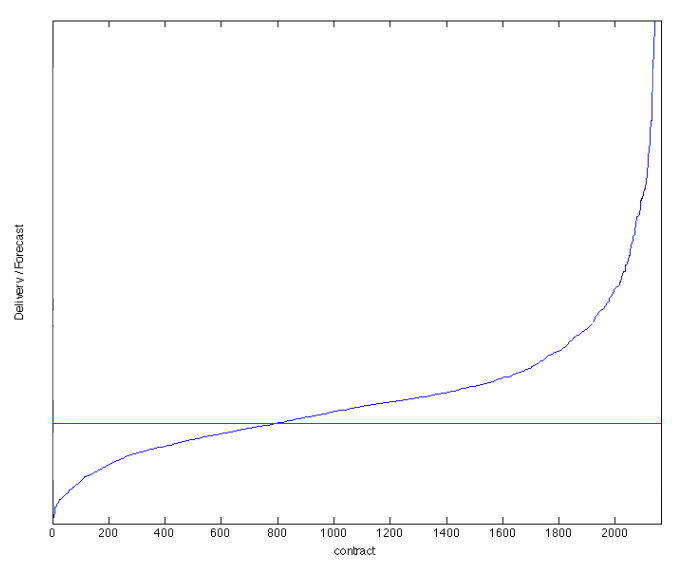

Figure 5: A distribution of supply forecast errors..

\subsubsection{Smoothness}

To improve the smoothness of delivery we consider the feedback system described in Section 4.4 We set the $\delta$ parameter to 4 hours, and $\beta$ - to 10 , implying that a contract that frontloads by more than 4 hours will get its delivery rate reduced to $10 \%$ of its original value. This effectively reduces the frontloading performed by the system. We denote this algorithm $\mathrm{HWM}+$, and show the results in Table 3

Table 3: Total delivery for Base and HWM approaches. Note that HWM+ obtains comparable smoothness to the baseline, while still drastically improving overall delivery.

\begin{tabular}{|l|c|c|c|}
\hline Algorithm & Delivery Improvement & $\sigma^{75}$ & $\sigma^{95}$ \\
\hline \hline Base & - & - & - \\
HWM & $53 \%$ & $+288 \%$ & $+634 \%$ \\
HWM + & $40 \%$ & $-66.0 \%$ & $+20.9 \%$ \\
\hline
\end{tabular}

Observe that HWM+ slightly decreased total delivey compared to HWM. However, it still dramatically improves delivery compared to the current baseline system, and its smoothness is on par - for the majority of the contracts the delivery is even smoother.

\subsubsection{Stress Test}

We have already observed that an accurate supply forecast is an integral component of the system. As we saw in Section 4.3 the HWM algorithm, when allowed to periodically re-optimize based on the latest delivery statistics, gracefully handles forecast errors. In this subsection, we empirically verify that the system indeed performs well even with large forecasting errors.

We altered the supply forecast to be biased towards underdelivery. To do this, we effectively doubled the supply forecast for all of the contracts. In this case, the supply forecasting system was over-predicting the available supply on more than three-quarters of the contracts. Although such a high supply forecasting error rate should be unlikely to occur in practice, it is a good demonstration of the system's robustness, even in adverse settings.

The results for the supply forecasting stress test are shown in Table 4 Note that since the current system, Base, does not use supply forecasting to make its serving decisions, its delivery is unaffected. It is not surprising that the overall delivery of HWM+ is lower; on the other hand, we observe that frequent reoptimization (the re-optimization happens 12 times per day) limits the impact of such high supply forecast errors. Moreover, even in such a stressed state, the $\mathrm{HWM}+$ algorithm performs better than Base.

Finally, just as we used a simple feedback system to remedy frontloading errors, we use the same system system to battle the underdelivery. Keeping $\delta$ at 4 hours, and $\beta$ - at 10 , we set $\beta+$ to 1.5 . In this scenario, when the delivery is 4 hours behind (with respect to the linear goal) we artificially increase the demand of the contract by $50 \%$. This increases the urgency on the contract, which leads it to deliver at a higher rate in the short term. Note that once the total delivery is within two cycles of the linear goal, the demand is no longer artificially boosted. We call this algorithm $\mathrm{HWM}++$ and show its results in Table 4 . This admittedly simple feed- 
back mechanism almost completely counteracts the effects of the supply forecast errors, resulting in near optimal delivery.

Table 4: Total delivery for the Base and HWM approaches. Note that $\mathrm{HWM}++$ obtains comparable smoothness to the baseline, while still drastically improving overall delivery.

\begin{tabular}{|l|c|c|c|}
\hline Algorithm & $\begin{array}{c}\text { Delivery } \\
\text { Imprv. }\end{array}$ & $\sigma^{75}$ & $\sigma^{95}$ \\
\hline \hline Base & - & - & - \\
HWM & $53 \%$ & $+288 \%$ & $+634 \%$ \\
HWM+ & $40 \%$ & $-66.0 \%$ & $+20.9 \%$ \\
HWM (2x SF errors) & $6 \%$ & $-5.0 \%$ & $+92.8 \%$ \\
HWM++ (2x SF errors) & $47 \%$ & $+2.6 \% \%$ & $+116 \%$ \\
\hline
\end{tabular}

\section{COMPARISON OF DUAL AND HWM}

We ran further experiments on DUAL and HWM, showing that the two perform comparably.

\subsection{Data}

We used a snapshot of actual data from two different time periods on two different areas of traffic to run our simulations. The first uses a week-long period of actual ad logs, downsampled at a rate of $10 \%$. We further used a set of real contracts active during that time period. The second also uses a week-long period of actual ad logs, but downsampled at a rate of $0.25 \%$. (The traffic on this area was much greater.)

\subsection{Experimental Results}

This experiment compares the HWM and DUAL algorithms, both with and without feedback. Our results report the delivery and smoothness for contracts that finished during the active time period, as well as the smoothness of the unfinished contracts during the active period. They are shown in Table 5, with ++ denoting algorithms with feedback. As we see, the general trend for smoothness is the same for both finished and unfinished contracts.

Table 5: Comparison of HWM and DUAL on the first data set

\begin{tabular}{|l|c|c|c|c|}
\hline Algorithm & $\begin{array}{c}\text { Delivery } \\
\text { Imprv. }\end{array}$ & $\begin{array}{c}\sigma^{75} \\
\text { (finished) }\end{array}$ & $\begin{array}{c}\sigma^{95} \\
\text { (finished) }\end{array}$ & $\begin{array}{c}\sigma^{75} \\
\text { (unfinished) }\end{array}$ \\
\hline \hline HWM++ & - & - & - & - \\
DUAL++ & $+4.82 \%$ & $+16.96 \%$ & $+2.44 \%$ & $+3.36 \%$ \\
HWM & $-43.89 \%$ & $+143.79 \%$ & $+367.30 \%$ & $+107.62 \%$ \\
DUAL & $-3.85 \%$ & $+173.16 \%$ & $+375.44 \%$ & $+126.89 \%$ \\
\hline
\end{tabular}

We also ran the comparison between HWM and DUAL on our larger data set. Here, we compare only the feedback algorithms. The results are shown in Table 6 .

Clearly, the algorithms perform much better, both in terms of under-delivery and smoothness, when using feedback. However, we see two somewhat surprising things. First, the under-delivery rate for both HWM and DUAL are comparable. In fact, HWM does somewhat better in the second
Table 6: Comparison of HWM and DUAL on the second data set

\begin{tabular}{|c|c|c|c|c|}
\hline Algorithm & $\begin{array}{c}\text { Delivery } \\
\text { Imprv. }\end{array}$ & $\begin{array}{c}\sigma^{75} \\
\text { (finished) }\end{array}$ & $\begin{array}{c}\sigma^{95} \\
\text { (finished) }\end{array}$ & $\begin{array}{c}\sigma^{75} \\
\text { (unfinished) }\end{array}$ \\
\hline \hline HWM++ & - & - & - & - \\
DUAL++ & $-11.79 \%$ & $+95.24 \%$ & $+155.99 \%$ & $+95.24 \%$ \\
\hline
\end{tabular}

data set. Note that both algorithms have very low underdelivery rates (although we cannot display the absolute values for confidentiality reasons), hence a difference of 5 or $10 \%$ translates to a very small absolute difference.

Second, DUAL is actually worse in terms of smoothness, despite the first term of its objective. Indeed, we see the first term of the DUAL objective (which attempts smoothness over all impressions) evaluates to a better value for the online DUAL allocation than the online HWM allocation. Yet the temporal smoothness that we report is worse.

Our belief is that this has three contributing factors: (1) The first term of the DUAL objective attempts smooth allocation across all impressions, with time being just one dimension among many (with others, like gender, age, interests, and so on being just as important), (2) HWM's firstcome-first-served approach tends to give many contracts very smooth allocation, with the contracts allocated near the end having potentially blocky distributions, while DUAL tends to distribute non-smoothness among all contracts, and (3) supply forecasting errors make it difficult for an optimal offline solution to translate to a truly smooth online allocation.

In general, many of the advantages of generating an optimal serving plan using a method like DUAL are mitigating because of forecasting errors and the feedback needed to correct for those errors. Thus, HWM provides a very practical solution in terms of speed and performance.

\section{RELATED WORK}

There are three major related problems in the context of guaranteed display advertising: the allocation problem, the booking problem, and the ad serving problem (the focus of this paper). In the allocation problem, the goal is to find an allocation of supply (user visits) and demand (contracts) so as to meet an optimization objective. In this context, Ghosh et al. 2009 and Yang and Tomlin 2008 propose various objectives to smooth the allocation of supply to demand so as to avoid the "lumpy" solutions characteristic of simple linear programming formulations Nakamura and Abe 2005. In the booking problem, the goal is to efficiently determine whether a new contract can be booked without violating the guarantees of existing booked contracts. The work of [Feige et al. 2008], Aleai et al. 2009, Negruseri et al. 2009 and Radovanovic and Zeevi 2009 consider various techniques and relaxations to solve this problem efficiently.

The guaranteed ad serving problem can be viewed as an online assignment problem, where some information is known about the future input, but the input is revealed in an online fashion one vertex at a time, and the goal is to optimize for an allocation objective. As such, this is related to the literature on online matching. In a celebrated result, Karp et al. 1990, showed that a simple randomized algorithm that 
finds a matching of size $n\left(1-\frac{1}{e}\right)$. However, Karp et al.'s solution only works for simple matching, requires maintaining counters across distributed servers, and does not apply to more general allocation objectives that are considered in display advertising. Feldman et al. Feldman et al. 2009 extend Karp et al.'s results and show that if some information about the future is available, the $n\left(1-\frac{1}{e}\right)$ bounds can be further improved. The proposed solution works by solving two problems offline, and using both solutions online. However, the entire solution graph, including both supply and demand nodes and the edges, has to be sent to the online server. Consequently, the plan as described is not compact, cannot be based on sampled user visits, and cannot generalize to unseen user visits - all of which are crucial requirements for guaranteed ad serving.

Another area of work that is related to the online allocation problem is two-stage stochastic optimization with recourse. In this model, the future arrival distribution is assumed to be known in advance, and the goal is to find an allocation that best matches the distribution. However, all of the algorithms (e.g., Katriel et al. 2007]) assume that the input is revealed in two stages, at first just the distribution of the future input, and then the full input is revealed. Consequently, these algorithms do not work in an online fashion, and the solution computed in the first stage does not generalize to unseen input.

The ad serving solution presented in this paper builds upon a mathematical result by Vee et al. Vee et al. 2010. that shows that for a fairly general set of allocation objectives, the optimal solution (except for sampling errors) can be compactly encoded as just the dual values of the demand constraints. Note that the approach of Devanur and Hayes Devenur and Hayes 2009, which also uses dual-based methods, does not apply in our setting since it cannot handle "at least" constraints.

We leverage the result of Vee et al. 2010 and propose a novel and efficient High Water Mark (HWM) algorithm that can be used to rapidly solve the allocation problem for large graphs and produce a compact, generalizable, ratebased allocation plan for a distributed ad server. Further, we introduce a rapid feedback loop, and show how this can form a theoretical basis for dealing with supply forecasting errors using the HWM algorithm. Finally, we also present an experimental evaluation of the proposed approach using real-world data.

\section{CONCLUSION \& FUTURE WORK}

We have presented a new ad serving system that relies on a compact allocation plan to decide which matching contract to show for every user visit. This plan-based approach is stateless, generalizable, allows for very high throughput and at the same time performs better than the current quotabased system. While the approach is sensitive to supply forecast errors, we have shown that frequent re-optimization helps correct supply forecast errors, and diminishes the impact that these errors have on delivery.

These rate-based algorithms show clear improvement in under-delivery for ad serving, while maintaining comparable smoothness. However, there is no clear winner between HWM and DUAL. Although DUAL performs better under perfect forecasts, its performance in practice is severely impacted by forecasting errors and feedback corrections. Thus,
HWM is quite attractive due to its lightweight implementation and speed, coupled with good overall performance.

\section{REFERENCES}

[Agarwal et al. 2010] Agarwal, D., Chen, D., Ji Lin, L., Shanmugasundaram, J., ANd Vee, E. 2010.

Forecasting high-dimensional data. In SIGMOD Conference, A. K. Elmagarmid and D. Agrawal, Eds. ACM, 1003-1012.

[Alaei et al. 2010] Alaei, S., Kumar, R., Malekian, A., AND VEE, E. 2010. Balanced allocation with succinct representation. In ACM SIGKDD Conference on Knowledge Discovery and Data Mining. 523-532.

[Aleai et al. 2009] Aleai, S., Arcaute, E., Khuller, S., Ma, W., Malekian, A., And Tomlin, J. 2009. Online allocation of display advertisements subject to advanced sales contracts. In Proc. of the 3rd Int. Workshop on Data Mining and Audience Intelligence for Advertising (ADKDD).

[Bharadwaj et al. 2010] BharadwaJ, V., Ma, W., Schwarz, M., Shanmugasundaram, J., Vee, E., Xie, J., AND YANG, J. 2010. Pricing guaranteed contracts in online display advertising.

[Devenur and Hayes 2009] Devenur, N. R. And Hayes, T. P. 2009. The adwords problem: online keyword matching with budgeted bidders under random permutations. In ACM Conference on Electronic Commerce. 71-78.

[Feige et al. 2008] Feige, U., Immorlica, N., Mirrokni, V., AND NAZERzAdeH, H. 2008. A combinatorial allocation mechanism with penalties for banner advertising. Presented at INFORMS.

[Feldman et al. 2009] Feldman, J., Mehta, A., Mirrokni, V. S., And Muthukrishnan, S. 2009. Online stochastic matching: Beating 1-1/e. In FOCS. IEEE Computer Society, 117-126.

[Ghosh et al. 2009] Ghosh, A., McAfee, P., Papineni, K., AND VAssilvitskis, S. 2009. Bidding for representative allocations for display advertising. In WINE, S. Leonardi, Ed. Lecture Notes in Computer Science Series, vol. 5929. Springer, 208-219.

[Karp et al. 1990] KarP, R. M., VAzIRANi, U. V., AND VAZIRANI, V. V. 1990. An optimal algorithm for on-line bipartite matching. In STOC '90: Proceedings of the twenty-second annual ACM symposium on Theory of computing. ACM, New York, NY, USA, 352-358.

[Katriel et al. 2007] Katriel, I., Kenyon-Mathieu, C., AND Upfal, E. 2007. Commitment under uncertainty: Two-stage stochastic matching problems. In ICALP, L. Arge, C. Cachin, T. Jurdzinski, and A. Tarlecki, Eds. Lecture Notes in Computer Science Series, vol. 4596. Springer, 171-182.

[Nakamura and Abe 2005] Nakamura, A. And Abe, N. 2005. Improvements to the linear programming based scheduling of web advertisements. J. Electronic Commerce Research 5, 75-98.

[Negruseri et al. 2009] Negruseri, C. S., Pasoi, M. B., Stanley, B., Stein, C., And Strat, C. G. 2009. Solving maximum flow problems on real world bipartite graphs. In $A L E N E X$, I. Finocchi and J. Hershberger, Eds. SIAM, 14-28. 
[Radovanovic and Zeevi 2009] Radovanovic, A. And ZeEvi, A. 2009. Revenue maximization in reservation-based online advertising. Presented at INFORMS.

[Vee et al. 2010] VeE, E., VAssilvitskit, S., AND Shanmugasundarum, J. 2010. Optimal online assignment with forecasts. Proc. of the ACM Conf. on Electronic Commerce.

[Yang and Tomlin 2008] YAng, J. And Tomlin, J. 2008. Advertising inventory allocation based on multi-objective optimization. Presented at INFORMS. 